\title{
On Learning and the Quality of Service in a Wireless Network
}

\author{
Tiina Heikkinen \\ Dept. of Mathematical Statistics \\ University of Lund, Box 118 \\ 22100 Sweden
}

\begin{abstract}
The purpose of this paper is to study the quality of service and optimal distributed resource allocation in a wireless network. A wireless network is similar to the Internet in that the system description must allow for distributed resource allocation under uncertainty. The quality of service that results from the operation of a distributed system is analyzed in terms of a signal-to-noise model of a power-controlled wireless network. The method of analysis is game theory with learninig. Recently, the allocation of resources in the Internet has been studies using solution concepts based on learnabilility. The learnability in the context of a distributed mobile network is studied here. By the appropriate redefinition of the strategy spaces of the users, the resource allocation game, defined between the users, is learnable in the sense that eventually the users learn the optimal strategy concerning resource usage.
\end{abstract}

Technical subject area: Wireless Communications, Quality of Service, Wireless Internet

\section{Introduction}

Future wireless multimedia networks provide new challenges to radio resource management. This paper concentrates on the managing of network resource allocation in a decentralized way. E.g. in packet-switched multimedia networks [1] users often send packets independently of each other. Recently [15] has illustrated how game theory can be used to formulate and analyze decentralized resource allocation in congested networks. The purpose of this paper is to discuss how game theory applies in the analysis of the distributed resource allocation of future multimedia networks, charatecterized by bursty transmissions across an arbitrarily time-varying channel (like the Internet). In particular, the learnability of the optimal strategies in a time-varying distributed environment is being analyzed.

This work studies the optimal quality of service $Q o S$ that can be achieved in a distributed wireless network and the mechanisms through which the optimal $Q o S$ can be reached under uncertainty. The mechanism analyzed in this work consists of a probabilistic learning automation. Under certain assumptions concerning the strategy spaces of the users (players in the network), the users learn the optimal strategies in a noisy

\footnotetext{
${ }^{0}$ Part of this work was carried out while the author was with Rutgers University.

G. Pujolle et al. (Eds.): NETWORKING 2000, LNCS 1815, pp. 679-688, 2000

(C) Springer-Verlag Berlin Heidelberg 2000
} 
asynchronous network model in bounded time. The general rapidness of convergence under asynchronous strategy choices of users remains an open issue.

By applying game theory under uncertainty, this paper seeks to study the dynamic allocation of resources in a wireless network in the case of uncertain time-varying link gain matrix. The model studied describes the uplink (the case of a downlink is analogous) of a wireless network that consists of $m$ users transmitting to $n$ receivers. The paper is organized as follows. Section 2 discusses solution concepts for the distributed dynamic resource allocation of a time-varying network. Section 3 presets a formal model of dynamic distributed resource allocation in a communication network. The extension of the model of distributed resource allocation to a mobile distributed network is discussed in section 4 . Section 5 concludes on implementation issues.

\section{Solution Concepts for a Wireless Network Under Uncertainty}

\subsection{Game Theory Under Uncertainty}

This paper discusses game theory under incomplete information in a setting where $n$ users share bandwidth in a time-varying network. Game theory under incomplete information refers to the situation prevalent in multimedia networks where some or all players (users) lack full information about some aspects of the game such as the other players payoff functions, or even their own payoff functions. This is the situation prevalent in future wireless multimedia networks where the system should operate in a distributed way under incomplete information [1].

The question then is, which are the appropriate solution concepts for a stochastic wireless network administered in a distributed fashion. As discussed in $[5,6]$ in the case of the Internet, traditional game-theoretical solution concepts need not apply in applications where economic agents know little, and new solution concepts such as those based on learning, are being needed.

There seems to be at least two strands to follow in the development of a new solution concept for future wireless multimedia, where Aumann's "common knowledge" can not be assumed to apply. First, following e.g. [5,6] a distributed system could be based on a learning approach (section 4). Second, various relaxations of the common knowledge assumption can yield new solutions [10].

\subsection{Distributed Resource Allocation in a Mobile System}

Resource allocation (transmit power allocation) in a wireless network has been studied as a deterministic centralized optimization problem and using stochastic approximation techniques [16]. This section discusses distributed solution concepts for resource management in distributed (decentralized) wireless networks under uncertainty.

Define the quality of service $(Q o S)$ in terms of signal-to-noise-ration $(S N R)$. The framework of analysis is the $S N R$ - model $[16,17]$ of a wireless network. Letting the users be the players and the signal-to-noise ratio of a user define the payoff of the user, the $S N R$-model defines an externality game [4]. To see this, first consider the definition of an externality game. 
Definition 1. A non-atomic game is a game with a continuum of users, in which no single player can affect the other players by changing only her own action. A nonatomic externality game (NAEG) is a nonatomic game with $m$ classes of players and submodular payoff functions [4].

Here maintain the assumption that no user can affect the other players by changing her action. Here the players are the users who ignore the impact of their choice of the use of resource (transmit power) on the externality (interference) of other users.

The key restriction on a representative user $i$ 's payoff (signal-to-noise ratio) $\alpha_{i}$ is that it only depends on her own strategy $x_{i}$ (transmit power) and the externality vector $f(x)$. Thus, define [16]

$$
\alpha(x, f)=\alpha_{i}\left(x_{i}, f(x)\right)=\frac{g_{i} x_{i}}{f(x)}=\frac{g_{i} x_{i}}{\sum_{j \neq i} g_{j} x_{j}}
$$

Here by definition $\alpha_{i}$ is a submodular function i.e. function with decreasing differences: for all $x, x^{\prime}$ in the strategy space and $x>x^{\prime}$,

$$
\alpha_{i}(x, f)-\alpha_{i}\left(x^{\prime}, f\right)=\frac{g_{i}\left(x-x^{\prime}\right)}{\sum_{j \neq i} g_{j} x_{j}}
$$

is nonincreasing (in fact decreasing) in $f$.

Thus, a wireless network is another example of a nonatomic externality game. Hence, learnability in a wireless network can be established by learnability conditions for an $N A E G$ [4]. The main result in [4] for simple $N A E G$ s connects their $O$-solvability (solvability by the elimination of overwhelmed strategies, ) to dominance- $D$-solvability and hence to best reply stability;

Definition 2. For a given player $i$, strategy $\mathbf{x}$ overwhelms strategy $\mathbf{x}^{\prime}$ relative to strategy set $S$ iff $\alpha_{i}(\mathbf{x}, f)>\alpha_{i}\left(\mathbf{x}^{\prime}, f^{\prime}\right)$ for all $f, f^{\prime}$. The set of strategies which are not overwhelmed are denoted by $O_{i}(S)$.

Define $O^{k}(S)=O\left(O^{k-1}(S)\right)$ with $O^{1}=O(S)$. Note $([4])$ that $O^{\infty}=\lim _{k \rightarrow \infty} O^{k}$ is well defined due to the monotonicity of $O$.

Definition 3. A game is $O$-solvable if card $\left(O^{\infty}\right)=1$ for almost all $i$.

$O$-solvability thus is a strong condition requiring that even in an asynchronous game where a player does not know the opponent's strategies, the player still can eliminate dominated strategies.

In order to apply solvability concepts for simple $N A E G$ s it is necessary to redefine the signal to noise model so that it becomes a simple $N A E G$, i.e. a participation game where players only have two choices, whether or not to participate (now). This redefinition is discussed in section 2.3 below and formalized in section 3 .

To motivate the application of solvability concepts for simple distributed $N E A G \mathrm{~s}$ in the context of a mobile distributed system assume that mobility as such does not change the nature of the underlying distributed externality game. Section 4 discusses the reasonability of this assumption. 


\subsection{Learning Games}

Following [5], the following optimization requirements can be placed on a probabilistic learning algorithm:

- When placed in an unknown but eventually stationary environment, the algorithm converges in bounded time, to strategies that maximize average payoff.

- Law of Effect: increasing the payoffs for a given strategy increases the probability of that the strategy being played in the future.

By assumption, for both the standard learning automation and responsive learning automation (a generalization of the former) there is a time $T$ after which there is a particular strategy whose payoffs are maximal for all histories of the play. For the power control game [10], this assumption does not directly apply, since the deterministic optimum strategy is a mixed strategy [10].

However, by redefining the strategy sets appropriately, the probabilistic learning algorithm becomes directly applicable in distributed resource allocation in a multimedia network, as demonstrated in section 3.2. Section 3.1 first constructs a delay-limited centralized solution to the congestion problem of sharing a limited bandwidth; by adding a temporal dimension, an atemporal solution concept for the congested wireless network can be made comparable to the temporal (delay-based) solution concepts to the congestion problem [15]. Section 3.2 extends 3.1 to a distributed setting.

\subsection{Mutual Knowledge Games}

A simple way to include imperfect information in the definition of an information capacity game between users is to define the information capacity as the outcome of a minimax game, following [10]. The minimax capacity allows to interpret the strategies as mixed strategies. The connection between mixed strategies and incomplete information games is discussed in [12] and the application of the connection to wireless networks in [10].

\section{A Delay-limited Solution to Resource Allocation}

\subsection{A Centralized Solution to Temporal Resource Allocation}

In a model with temporal dynamics, the objective is, in a discretized time model, to maximize, with respect to transmit powers, the service quality as measured by the signal to noise ratio $(S N R) \alpha$ at each time interval, taking into account a delay cost formalized below following [8]. A delay-limited capacity is related to the informationtheoretic capacity and has recently also been discussed in [2]. A delay-limited capacity has an economic flavor; in [8] and [2] a certain threshold level capacity must be achieved; otherwise the usage of transmit power would be inefficient. Intuitively, when fading is very bad is is better to accept outage [2]; this idea is formalized below following [8].

Here, like in [14], the different rates are modeled by varying the elements in the gain matrix $\mathbf{G}(m \times n$ or $m \times n m$, where $m$ is the number of information sources and 
$n$ is the number of receivers). In the generalized multiple access model (see appendix C), the coefficient $g_{i, j}=(\mathbf{G})_{i, j}$ denotes the fading of transmitted information signals by user $i$ to receiver $j$ that accommodates transmissions of datatype $j$.

Denote the solution sequence by $\left\{\alpha_{t}^{*}\right\}_{t=0}^{T}$. Formally, this sequence is the solution for (when taking into account the delay cost, formalized in (4) below

$$
\max E \sum_{t=0}^{T} \alpha_{t} \quad \text { s.t. } \quad \mathbf{F}_{t} \mathbf{x}_{t} \alpha_{t} \leq \mathbf{G}_{t} \mathbf{x}_{t}
$$

Each period the optimal capacity $\alpha$ is the solution to Problem 1: maximize $\alpha$ with respect to $\mathbf{x}$ subject to $\mathbf{x} \geq 0, \mathbf{x} \leq \mathbf{x}_{\max }$ and subject to the constraint, stating the $Q o S$ requirement, $\mathbf{G x}-\alpha \mathbf{F x} \geq 0$. The optimal ray solution to the one period problem is given by $x_{i}=\frac{1}{g_{i}}$ and $p_{i}=\frac{1}{n} \forall i$. To model dynamic resource allocation, introduce a parameterized discount delay cost $0 \leq b \leq 1$ and an additive delay cost $c \geq 0$. Let there be two state variables, $\alpha$ and $i: i=0$ if $\alpha$ is rejected in the beginning of the period $\mathrm{t}$ (i.e. $\alpha<\bar{\alpha}), i=1$ if $\alpha$ is accepted: $\alpha \geq \bar{\alpha}$. The Bellman equations for $v(\alpha, i), i=0,1$ are, assuming there is in addition to the delay cost defined by $c$ below a proportional (to the value function) delay cost component $b \in(0,1)$ when deferring the choice of the power vector by $\Delta(t)$ to period $t+1$ with different realizations: $E\left(\mathbf{F}_{t+1}\right)$ and $E\left(\mathbf{G}_{t+1}\right)$ :

$$
\begin{aligned}
& v(\alpha, 0)=\max \{\alpha+b E v(\alpha, 1), b E v(y, 0)-c\} \\
& v(\alpha, 1)=\max \{\alpha+b E v(y, 0)\} .
\end{aligned}
$$

Define $\bar{\alpha}$ by

$$
\bar{\alpha}+b E v(\bar{\alpha}, 1)=b E v(y, 0)-c,
$$

where $\bar{\alpha}$ is the reservation (i.e. threshold) capacity. Choose the foregoing model to define the expected value of capacity as [8]:

$$
E v(\alpha, 0)=\frac{\bar{\alpha}(1+b)}{b(1-b)}+\frac{c}{b(1-b)} .
$$

$E v(\alpha, 0)$ captures the optimal dynamic behavior of the network, since its arguments satisfy the Bellman equation.

\subsection{Learnability in a Decentralized System}

The above section assumed that the distribution $f$ of $\alpha=\frac{1}{m-1}$ is stationary. However, this need not be the case for a bursty time-varying multimedia network. A solution concept based on responsive learning automata is discussed next.

Following [5] consider a discrete-time environment with $M$ possible strategies. Let $r_{i}^{t}$ denote the payoff (capacity) for playing strategy $i$ at time $t$, where as above, $0 \leq$ $r_{i}^{t} \leq 1$. The state of an automation is a probability vector such that at time $t$ the automation picks strategy $i$ with probability $p_{i}^{t}$ at random. To begin with, assume a stationary environment like in section $A$ and consider a standard learning automation $L A$ (generalized by a responsive one.) A standard parameterized (by $\beta$ ) updating rule $[5]$ is 


$$
\begin{gathered}
p_{i}^{t+1}=p_{i}^{t}+\beta r_{i}^{t}\left(1-p_{i}^{t}\right) \\
\forall j \neq i p_{j}^{t+1}=p_{j}^{t}\left(1-\beta r_{i}^{t}\right)
\end{gathered}
$$

Assuming the $r_{i}^{t}$ are chosen from some stationary probability distribution so that $P\left(r_{i}^{t} \leq x\right)$ is given by $F_{i}(x)$ independent of $t$. The $L A$ s are $\epsilon$-optimal in that for any $\epsilon>0$ there exists some $\beta$ such that

$$
\lim _{t \rightarrow \infty} E\left(\sum_{i=1}^{m} p_{i}^{t} r_{i}^{t}\right)>\max \left(E\left(r_{i}^{s}\right)\right)-\epsilon
$$

Here consider $M=2$ possible strategies: accept $A$ or reject $R$ (and carry a delay cost parameterized by $(c)$ as above). Then $r_{i}^{t}=r^{t}=\frac{1}{m^{t}-1}$, where $V$ here is a constant, and the standard updating rule is given by, if strategy $A$ is picked at time $t$

$$
\begin{gathered}
p_{A}^{t+1}=p_{A}^{t}+\beta \frac{1}{m^{t}-1}\left(1-p_{A}^{t}\right) \\
p_{R}^{t+1}=p_{R}^{t}\left(1-\beta \frac{1}{m^{t}-1}\right)
\end{gathered}
$$

Notice that here the payoffs are defined, based on the analysis in 3.1. Then the learning automation defined by (10)-(11) is $\epsilon$-optimal [5]: the automation can achieve an asymptotic expected payoff arbitrarily close to the optimal payoff. The play converges to a single strategy.

In an arbitrarily time-varying environment, the collapse to a single strategy is not necessarily reasonable. A responsive learning automation $R L A$ would require that all strategies are played with strictly positive probabilities:

$$
\begin{aligned}
& p_{A}^{t+1}=p_{A}^{t}+\beta \frac{1}{m^{t}-1} a_{R}^{t} p_{R}^{t} \\
& p_{R}^{t+1}=p_{R}^{t}-\beta \frac{1}{m^{t}-1} a_{R}^{t} p_{R}^{t}
\end{aligned}
$$

where $a_{i}^{t}=\min \left\{1, \frac{p_{i}^{t} \beta / 2}{\beta p_{i}^{t}}\right\}$. A more general convergence criterion for $R L A \mathrm{~s}$ than $\epsilon$-convergence is given in [5]. Loosely, convergence for $R L A \mathrm{~s}$ is defined so that the probability of playing nonoptimal strategies converges to zero.

\section{Learnability on a Mobile Distributed Network}

Assume the environment becomes stationary after some finite time. Then, as argued in [4], a simple $N A E G$ such as defined above (by $M=2$ ) is $O$-solvable which implies that reasonable learners will converge to the unique Nash-equilibrium even in noisy distributed asynchronous settings. 
In particular, showing that the participation game defined above is best-reply $B R$ stable is sufficient for a simple $N A E G$ to be $O$-solvable and thus learnable. This can be done by checking $D$-solvability of the participation game: an externality game is $B R$ stable if and only if it is $D$-solvable, i.e. dominance-solvable: solvable by the iterated elimination of dominated strategies [4].

Definition 4. For a player $i$, pure strategy $x$ dominates pure strategy $x^{\prime}$ iff $\alpha_{i}(x, f)>$ $\alpha_{i}\left(x^{\prime}, f\right) \forall f$.

Definition 5. Let $D^{\infty}$ denote the set of strategies after infinite iterative elimination of dominated strategies. A game is $D$-solvable if card $\left(D^{\infty}\right)=1$ for almost all $i$.

If the utility functions of the users are similar (users value the trade-off between delay and capacity similarly), the users are symmetric with respect to one another in the participation game, and it suffices to study a representative user. An analogous result to Theorem 1 in [4] follows:

Proposition 1. A wireless network as defined as a participation game between users is D-solvable with a unique Nash-equilibrium.

Proof. A strategy profile $s$ is a Nash equilibrium in pure strategies if for almost all users $i$ and all $z \alpha_{i}(s(i), f(s)) \geq \alpha_{i}(s(z), f(s))$. Given the number of users in the system $m$, to accept is the unique best reply for a representative user if $\frac{1}{m-1} \geq \bar{\alpha}$, where $\bar{\alpha}$ only depends on given parameters (see (5)).

The direct applicability of learnability for distributed systems to mobile distributed systems can be based on viewing the mobile system as intrinsically distributed system; a rationale for this as given in [9] is that formally, the distributed resource allocation problem for a wireless system is very similar to the distributed resource allocation problem for the Internet [11].

The main difference between the resource allocation model for the Internet and the model for the mobile system is that in the latter mobility is taken into account by the variable link coefficients $0 \leq g_{i j} \leq 1$ between user $i$ and base $j$ whereas in the former these coefficients are binary variables. Assuming for simplicity the binary case for the mobile distributed system would be sufficient to guarantee that users with similar utility functions agree with the optimal capacity equalization $\alpha_{i}=\alpha$; letting each user $i$ have the choices to transmit or not, i.e. $x_{i}=0$ or $x_{i}=1$, is then equivalent to the choices being accept or reject the resulting capacity $\frac{1}{m-1}$, as above.

Also, in general, when the $g_{i j}$ 's are not binary variables, the distributed resource allocation game implies capacity equalization if the user optimize utility functions $u(X)$ with respect to the received signal $X=g x$ instead of with respect to the energy usage $x$. Then again there are two choices at each time period: $X_{i}=X^{*}=u^{\prime-1}(0) \forall i$ or $X_{i}=0 \forall i$. In conclusion, learnability for a mobile distributed network can be in principle similarly defined as for a distributed network. 


\section{Conclusion: On Implementation Issues}

This paper has introduced a framework for the analysis of distributed resource allocation under uncertainty. The paper studied distributed power control in a dynamic wireless network. A solution based on responsive learning automata was suggested.

A probabilistic learning automation is based on a randomized algorithm that can be unlucky, but eventually converges. The two simulation studies commented in [6] are encouraging but not conclusive and can be summarized as follows. The first work on cost sharing games is due to Chen [3] whose results show rapid and robust convergence to the unique Nash-equilibrium in the $O$-solvable case. The $D$-solvable game, however, was strongly affected by asynchrony and showed less robust convergence. Second, simulations on network games reported by Greenberg, Friedman and Shenker [7] also observed convergence. The discussion in section 4 suggests that these results can be applicable to a mobile distributed network but more simulations are needed to understand the relevance of solution concepts based on learning for a distributed wireless system.

\section{Appendix A}

Let $M^{m} \equiv\left\{\mathbf{x} \in \Re_{+}^{m} \mid \sum_{i} x_{i}=1\right\}$. Second, define the $n-1$ simplex: $M^{n} \equiv\{\mathbf{p} \in$ $\left.\Re_{+}^{n} \mid \sum_{i} p_{i}=1\right\}$.

The number $\alpha$ is defined by

$$
\alpha=\max _{\mathbf{x} \in M^{m}} \min _{\mathbf{p} \in M^{n}} \frac{\sum_{j=1}^{n}\left[\sum_{i=1}^{m} g_{i j} x_{i}\right] p_{j}}{\sum_{j=1}^{n}\left[\sum_{i=1}^{m} f_{i j} x_{i}\right] p_{j}}
$$

\section{Appendix B}

Solving for the optimal threshold $\bar{\alpha}$ gives the following characterization ([8]):

$$
\bar{\alpha}=\frac{E(\alpha)-\int_{0}^{\bar{\alpha}} \alpha f(\alpha) d \alpha-\frac{c}{b}}{\frac{1}{b}+(1-F(\bar{\alpha}))} .
$$

where $F$ is the cumulative density function of $\alpha$. In the absence of the discount cost $b$ a one perid approximation of $\bar{\alpha}$ is given by

$$
\bar{\alpha}=\frac{E(\alpha)-\int_{0}^{\bar{\alpha}} \alpha f(\alpha) d \alpha-\frac{c}{\tau}}{(1-F(\bar{\alpha}))},
$$

where $\tau=T-t$ and capacity $\alpha_{t}$ is accepted at time $t$.

The expression (16) gives a one-time-stopping rule; applying this repeatedly over time approximates the optimal dynamic resource allocation. 


\section{Appendix C}

The key features of a wireless network can be captured using a fairly elementary mathematical model. Typically, both the receivers and transmitters are scattered in some geographical area and the transmitted signals propagate through a physical channel modeling the attenuation of transmitted signal powers through each propagation path. The multiple access protocol defines, together with channel models, the associated interference model. Often interference is modeled as Gaussian noise, which is justified if there are numerous interferes with pseudo-random waveforms and if they are sufficiently weak when compared to the desired user [13].

Uplink

Here we summarize the uplink power control problem and characterize the optimal solution. Consider first an isolated cell with one antenna. We assume that the received signal $\mathbf{z}$ can be described by a linear model

$$
z_{i}(t)=\sum_{j=1}^{m}\left(x_{j} g_{i j}\right)^{\frac{1}{2}} b_{j} s_{j}+n,
$$

where $n$ is the additive Gaussian noise, $b_{j}$ denotes information bit transmitted by user $j$ and $s_{j}$ is the signature waveform of user $j$ [16]. The power control problem is to $\min \sum x_{i}$ such that

$$
\mathbf{G} \mathbf{x}=\alpha(\mathbf{F} \mathbf{x}+\mathbf{n}),
$$

where $\mathbf{F}$ denotes the interference (externality) matrix. In the absence of external noise, the optimal solution can be characterized as in appendix A [8].

\section{References}

1. F. Borgonovo and M. Zorzi, "Slotted Aloha and CDPA: A Comparison of Channel Access Protocols in Cellular Systems, Wireless Networks", 3, 1997

2. G. Caire, G. Taricco and E. Biglieri, "Optimum Power Control Over Fading Channels", IEEE Transactions on Information Theory, 41, 5, 1999

3. Y. Chen, "Asynchronicity and Learning in Cost Sharing Mechanisms", Mimeo 1996

4. E. Friedman, "Learnability in a Class of Non-Atomic Games arising on the Internet", Mimeo 1998

5. E. Friedman and S. Shenker, "Synchronous and Asynchronous Learning by Responsive Learning Automata", Mimeo 1996

6. E. Friedman and S. Shenker, "Learning and Implementation on the Internet", Mimeo 1998

7. A. Greenwald, E. Friedman and S. Shenker, "Learning in Network Contexts: Experimental Results from Simulations", Mimeo 1998

8. T. Heikkinen, "Capacity and Resource Allocation in a Multi-Media network", IEEE International Symposium on Information Theory, Boston MA 1998

9. T. Heikkinen, "Optimal Quality of Service and Pricing in the Wireless Internet", proc. ITC Specialist Seminar on Mobile Systems, Lillehammer, March 2000

10. T. Heikkinen, "A Minimax Game of Power Control Under Incomplete Information", DIMACS Technical Report 99-43 
11. F. Kelly and R. Gibbens, "Resource Pricing and the Evolution of Congestion Control", http://www.statslab.cam.ac.uk/ frank/evol.html

12. M. Osborne and A. Rubinstein, A Course in Game Theory, MIT Press 1994

13. T. Rappaport, Wireless communications, IEEE Press, 1996

14. A. Sampath, P. Kumar and J. Holtzman, "Power Control and Resource Management for a Multimedia CDMA Wireless System", proceedings of PIMRC 1995, Toronto, Canada

15. S. Shenker, "Making Greed Work in Networks", IEEE Transactions on Networking, 3(6), 1995

16. S. Ulukus and R. Yates, "Stochastic Power Control for Cellular Radio Systems", IEEE Transactions on Communications 46(6), 1998

17. Zander, J., "Performance of Optimum Transmitter Power Control in Cellular Radio Systems", IEEE Transactions on Vehicular Technology, vol. 41, no. 1, Feb. 1992 